\title{
Neuropeptide S Receptor Induces Neuropeptide Expression and Associates With Intermediate Phenotypes of Functional Gastrointestinal Disorders
}

(Gastroenterology 2010;138:98-107)

Jung Mook Kang, MD, PhD

Department of Internal Medicine, Healthcare Research Institute, Seoul National Universtiy Hospital Healthcare System Gangnam Center, Seoul, Korea

\section{Summary}

Irritable bowel syndrome (IBS) is the most common gastrointestinal disorder, of largely unknown etiology and pathobiology. There is growing evidence regarding the genetic contribution in IBS, however the precise etiology of IBS is still unknown. Recently, it has been proposed that several genetic markers are associated with some aspect of IBS. Neuropeptide S receptor 1 (NPSR1), the receptor for neuropeptide S (NPS), is expressed on the intestinal epithelium, and is involved in inflammation, anxiety, and nociception. NPSR 1 gene was recently found to be genetically associated with inflammatory bowel disease and asthma. ${ }^{1,2}$ The author wanted to determine whether NPS induces expression of gastrointestinal (GI) neuropeptides; and to associate NPSR1 single nucleotide polymorphisms (SNPs) with symptom phenotype and GI functions in health and functional GI disorders (FGID).

The author undertook the present study in vitro and in vivo model together. First, the effect of NPS on messenger RNA expression of neuropeptides was assessed in NPSR1-tranfected HEK293 cells. Second, 17 NPSR 1 polymorphisms were compared between 466 FGID patients and 233 healthy controls. They showed that NPS-NPSR1 signaling induced increased expressions of cholecystokinin, vasoactive intestinal peptide, peptide YY, and somatostatin. There were no significant associations with phenotypes of FGID symptoms. However, there were several NPSR1 SNPs associated with individual motor or sensory functions; the associations of SNPs rs2609234, rs6972158, and rs1379928 with colonic transit rate, while the rs1379928 polymorphism was also associated with pain, gas, and urgency sensory ratings at $36 \mathrm{~mm} \mathrm{Hg}$ distention. The author concluded that the expression of several neuropeptides is induced upon NPS-NPSR1 signaling and NPSR 1 variants are associated with colonic transit in FGID.

Received: March 23rd, 2010 Accepted: March 30th, 2010

(c) This is an Open Access article distributed under the terms of the Creative Commons Attribution Non-Commercial License (http://creativecommons. org/licenses/by-nc/3.0) which permits unrestricted non-commercial use, distribution, and reproduction in any medium, provided the original work is properly cited.

*Correspondence: Jung Mook Kang, MD, PhD

Department of Internal Medicine, Healthcare Research Institute, Seoul National Universtiy Hospital Healthcare System Gangnam Center, 39th FL., Gangnam Center 737, Yeoksam-dong, Gangnam-gu, Seoul 135-984, Korea

Financial support: None.

Tel: +82-2-2112-5749, Fax: +82-2-2112-5635, E-mail: jungmook2000@yahoo.co.kr

Conflicts of interest: None. 


\section{Comment}

IBS is a multifactorial disorder, and several patholphysiological mechanisms have been proposed, including altered bowel motility, visceral hypersensitivity, psychosocial factors, imbalance in neurotransmitters, and infections. ${ }^{3}$ Familial aggregation and twin studies suggest that genetic factors are thought to have a role in the manifestation of functional dyspepsia and IBS. ${ }^{4-10}$ Recent studies have noticed the importance of polymorphisms in the promoter region of the serotonin reuptake transporter gene, G-protein, cholecystokinin receptor, and cytokines such as IL-10. ${ }^{11,12}$

NPS is a recently identified bioactive 20 amino acid peptide whose primary sequence is highly conserved in different species. ${ }^{13}$ NPS selectively binds and activates an orphan G-protein coupled receptor, named NPSR $1 .{ }^{13}$ The biological function of the NPSR1-NPS system in the gut is still poorly understood. Previous studies have reported NPSR1 expression in epithelial cells of several organs and tissues, and an increase of this expression during inflammations, such as IBS and asthma. ${ }^{1,2}$ Thus, it may conceivably be associated with IBS given the increasing evidences of association of minor inflammation or prior infection with IBS. Recently, inhibition of distal colonic transit has been shown in mice upon intracerebroventricular administrations of NPS. ${ }^{14}$

In this study, the expression of several neuropeptides (cholecystokinin, vasoactive intestinal peptide, peptide YY, and somatostatin) involved in the control of physiological motor and sensory functions in gastrointestinal tract increase upon NPS-NPSR1 signaling in an in vitro model. These finding suggest that genetic alterations affecting NPSR1 expression might result in excessive or diminished neuropeptide inductions leading to perturbed gut function and possibly FGID. In these aspects, the authors have also studied 17 NPSR1 SNPs in an ethnically homogeneous group of 466 FGID patients with Rome II-positive criteria and 233 healthy controls from one geographical region of the United States. At present, most studies that investigated the genetic factors associated with FGID have been carried out in patients with IBS. Although patients with functional dyspepsia were included in this study, main population of FGID was IBS patients occupying about $90 \%$. The author failed to demonstrate significant associations of NPSR1 genotypes with symptom phenotypes of FGIDs. Meanwhile, intermediate phenotypes related to motor or sensory function were associated with 3 NPSR1 SNPs (rs2609234, rs6972158, and rs1379928).
Although the impacts of these SNPs on colonic transit rate were relatively weak with 10-16\% accelerations of GC (geometric center) 24/48 hours, these finding suggest that these polymorphisms could be related to diarrhea-predominant IBS. In addition, the rs1379928 polymorphism was also associated with increases of pain, gas, and urgency sensory ratings at $36 \mathrm{mmHg}$ distention to around 9.9-22.5\%. However, in electrophoretic mobility shift assays on 3 different cell lines, the author failed to demonstrate different bindings of nuclear proteins to DNA sequences according to rs1379928 alleles which affect both the colorectal motor and sensory function. Therefore further studies are needed to assess whether this SNP is truly functionally relevant. Their results provide evidences for involvement of NPSR 1 in the genetic susceptibility to intermediate phenotypes of gastrointestinal function and represent at least one potential mechanism to explain the genetic associations with IBS.

Main focus of this study is not on symptom phenotypes but rather on physiological phenotypes, thus, direct clinical relevance is unclear. However, these are also the strengths of the study because intermediate phenotypes could be more clearly defined and measured than symptom phenotype. In conclusion, this study provides the first evidence of an association of NPSR1 polymorphisms with gastrointestinal motor and sensory functions that are relevant to IBS. Replication studies will be important to confirm these findings in independent populations. Further analyses of NPSR1 function are encouraged to elucidate its role in FGIDs and the potential associations with epithelial barrier functions, inflammation, sensation, transit, and satiation in health and disease.

\section{References}

1. Laitinen T, Polvi A, Rydman P, et al. Characterization of a common susceptibility locus for asthma-related traits. Science 2004;304: 300-304.

2. D'Amato M, Bruce S, Bresso F, et al. Neuropeptide s receptor 1 gene polymorphism is associated with susceptibility to inflammatory bowel disease. Gastroenterology 2007;133:808-817.

3. Horwitz BJ, Fisher RS. The irritable bowel syndrome. N Engl J Med 2001;344:1846-1850.

4. Kalantar JS, Locke GR 3rd, Zinsmeister AR, Beighley CM, Talley NJ. Familial aggregation of irritable bowel syndrome: a prospective study. Gut 2003;52:1703-1707.

5. Levy RL, Jones KR, Whitehead WE, Feld SI, Talley NJ, Corey LA. Irritable bowel syndrome in twins: heredity and social learning both contribute to etiology. Gastroenterology 2001;121:799-804.

6. Saito YA. Genes and irritable bowel syndrome: is there a link? Curr Gastroenterol Rep 2008;10:355-362. 
7. Camilleri M, Carlson $\mathrm{P}$, Zinsmeister AR, et al. Mitochondrial DNA and gastrointestinal motor and sensory functions in health and functional gastrointestinal disorders. Am J Physiol Gastrointest Liver Physiol 2009;296:G510-G516.

8. Kim HJ, Camilleri M, Carlson PJ, et al. Association of distinct alpha(2) adrenoceptor and serotonin transporter polymorphisms with constipation and somatic symptoms in functional gastrointestinal disorders. Gut 2004;53:829-837.

9. Andresen V, Camilleri M, Kim HJ, et al. Is there an association between GNbeta3-C825T genotype and lower functional gastrointestinal disorders? Gastroenterology 2006;130:1985-1994.

10. Camilleri CE, Carlson PJ, Camilleri M, et al. A study of candidate genotypes associated with dyspepsia in a U.S. community. Am J
Gastroenterol 2006;101:581-592.

11. Adam B, Liebregts T, Holtmann G. Mechanisms of disease: genetics of functional gastrointestinal disorders - searching the genes that matter. Nat Clin Pract Gastroenterol Hepatol 2007;4:102-110.

12. Park SY, Rew JS, Lee SM, et al. Association of CCK 1 receptor gene polymorphisms and irritable bowel syndrome in Korean. J Neurogastroenterol Motil 2010;16:71-76.

13. Okamura N, Reinscheid RK. Neuropeptide S: a novel modulator of stress and arousal. Stress 2007;10:221-226.

14. Han RW, Chang M, Peng YL, et al. Central Neuropeptide S inhibits distal colonic transit through activation of central Neuropeptide $\mathrm{S}$ receptor in mice. Peptides 2009;30:1313-1317. 